ИЗВЕСТИЯ АКАДЕМИИ НАУК ЭСТОНСКОЙ ССР. ТОМ 30 ФИЗИКА * МАТЕМАТИКА. 1981, № 1

\title{
ЗАВИСИМОСТЬ МЕЖМОДОВОГО РАССТОЯНИЯ GaAs-AlGaAs- ГЕТЕРОЛАЗЕРА ОТ ПАРАМЕТРОВ РЕЗОНАТОРА
}

\author{
(Представил К. К. Ребане)
}

Спектральное расстояние между соседними линиями $\Delta \lambda$ в спектре излучения полупроводникового лазера может быть определено формулой

$$
\Delta \lambda=\lambda^{2} / 2 L \bar{n},
$$

где $\lambda$ - длина волны, $\quad \bar{n}=n-\lambda \frac{d n}{d \lambda} \quad$-эффективный показатель преломления (ЭПП), $n$ - показатель преломления вещества, заполняющего резонатор, и $L-$ длина резонатора. Приведенная формула вытекает из интерференционного условия для резонатора Фабри-Перо.

K гомолазерам эта формула применима непосредственно, так как излучение распространяется здесь в веществе, показатель преломления которого можно считать в первом приближении постоянной величиной, совпадающей с таковой для материала, из которого лазер изготовлен $\left[{ }^{1}\right]$.

В гетеролазерах обстановка сложнее, так как некоторая доля излучения распространяется в широкозонных эмиттерах. Поэтому межмодовое расстояние зависит здесь не только от показателя преломления активной среды, но и от показателя преломления эмиттеров, а также от толщины активного слоя. Попытка установить эту зависимость сделана в $\left[{ }^{2}\right]$, где выведена приближенная формула для межмодового расстояния гетеролазера. Однако в силу принятых в $\left[{ }^{2}\right]$ приближений эта формула неприменима для GaAs-AlGaAs-гетеролазеров.

Целью настоящего исследования является выяснение зависимости межмодового расстояния от параметров резонатора GaAs-AlGaAsгетеролазеров.

\section{Теоретическое рассмотрение}

Межмодовое расстояние для гетеролазера может быть определено из зависимости постоянной распространения $(\gamma)$ (см., напр., $\left[{ }^{3}\right]$ ) от длины волны. При этом следовало бы учитывать две зависимости показателя преломления от координаты: во-первых, весьма резкую в направлении, перпендикулярном плоскости $p$-n-перехода (возникающую из-за различия материалов активного слоя и эмиттеров), и, во-вторых, весьма слабую в плоскости $p$ - $n$-перехода (возникающую из-за неоднородности тока инжекции в полосковом лазере). Однако результаты анализа в [4] показывают, что постоянная распространения $\gamma$ определяется в 
основном первой из названных зависимостей. Вторая же зависимость приводит (в условиях, близких к реальным), по данным $\left[{ }^{4}\right]$, лишь к незначительным уточнениям величнны $\gamma$. Так, если расчет вести до третьей низшей поперечной моды, то уточнение составит не более $0,1 \%$.

На основе этих данных мы можем при расчете постоянной распространения ограничиться моделью трехслойного диэлектрического * волновода $\left[{ }^{3,5}\right]$, удобной с точки зрения упрощения расчетов. Определение постоянной распространения для ТЕ моды в таком волноводе сводится $[3,5]$ к решению трансцендентного уравнения

$$
\tan Q D=Q\left(\sqrt{1-Q^{2}}+\sqrt{\eta-Q^{2}}\right) /\left(Q^{2}-\sqrt{\left(1-Q^{2}\right)\left(\eta-Q^{2}\right)}\right)
$$

относительно $Q$. В этом уравнении $\eta$ характеризует асимметрию волновода, $D$ является приведенной толщиной волновода, а $Q$ - внутренним поперечным параметром, который устанавливает распределение электромагнитного поля в волноводе $\left[{ }^{3,5}\right]$. Постоянная распространения вычисляется при известном $Q$ по формуле

$$
\gamma^{2}=k^{2}\left(\varepsilon_{2}-Q^{2}\left(\varepsilon_{2}-\varepsilon_{1}\right)\right),
$$

где $k$ - волновой вектор в свободном пространстве, $\varepsilon_{2}$ - диэлектрическая постоянная активного слоя и $\varepsilon_{1}$ - бо́льшая из диэлектрических постоянных эмиттеров. Для численного решения приведенного трансцендентного уравнения необходимо знать величины показателей преломления для всех трех слоев. Наиболее полные данные о показателе преломления $\mathrm{Al}_{x} \mathrm{Ga}_{1-x} \mathrm{As}$ опубликованы в [ $\left.{ }^{7}\right]$. Они относятся к материалу, имеющему $n$-тип проводимости. Зависимость показателя преломления от длины волны излучения для образцов GaAs c различной концентрацией свободных электронов и дырок приведена в $\left[{ }^{8}\right]$. Данные последней работы позволяют сделать вывод. что образцы GaAs с концентрацией электронов и дырок менее $2 \cdot 10^{18} \mathrm{~cm}^{-3}$ имеют весьма близкие значения показателя преломления, если ограничиться рассмотрением длинноволновой области спектра, включая и область генерации (т. е. $\varepsilon<1,40$ эB). Можно думать, что это свойство остается в силе и для $\mathrm{Al}_{x} \mathrm{Ga}_{1-x}$ As. Такое предположение оправдывает использование данных из $\left[{ }^{7}\right]$ для эмиттеров и активного слоя (содержащих $\mathrm{Al}$ ) исследуемых в настоящей работе гетеролазеров, так как концентрация свободных носителей в последних не выходит из указанных выше пределов.

Таким образом, протабулировав данные $[7,8]$, мы смогли рассчитать постоянную распространения в зависимости от длины волны для гетеролазеров, имеющих различные толщины активного слоя и содержаших различные концентрации Al в эмиттерах и в активном слое.

$\mathrm{Ha}$ основе рассчитанной величины постоянной распространения ** были установлены (по аналогии с однородными средами) показатель преломления гетеролазеров $n^{*}=\gamma / k$ и ЭПП гетеролазеров $\bar{n}^{*}=n^{*}-\lambda \frac{d n^{*}}{d \lambda}$. Эти величины соответствуют некоторой гипотетической однородной среде, излучение в которой имеет такую же постоянную распространения, как и в реальном гетеролазере. Введение таких величин в качестве характеристик гетеролазеров делает более удобным сравнение параметров гетеролазера с параметрами материалов, из ко. торых он изготовлен.

* Учет усиления в активной среде и поглощения в эмиттерах приводит также к несущественным поправкам [ $\left.{ }^{6}\right]$.

** Расчет был проведен на ЭВМ М-4030 Института фнзики АН ЭССР. 

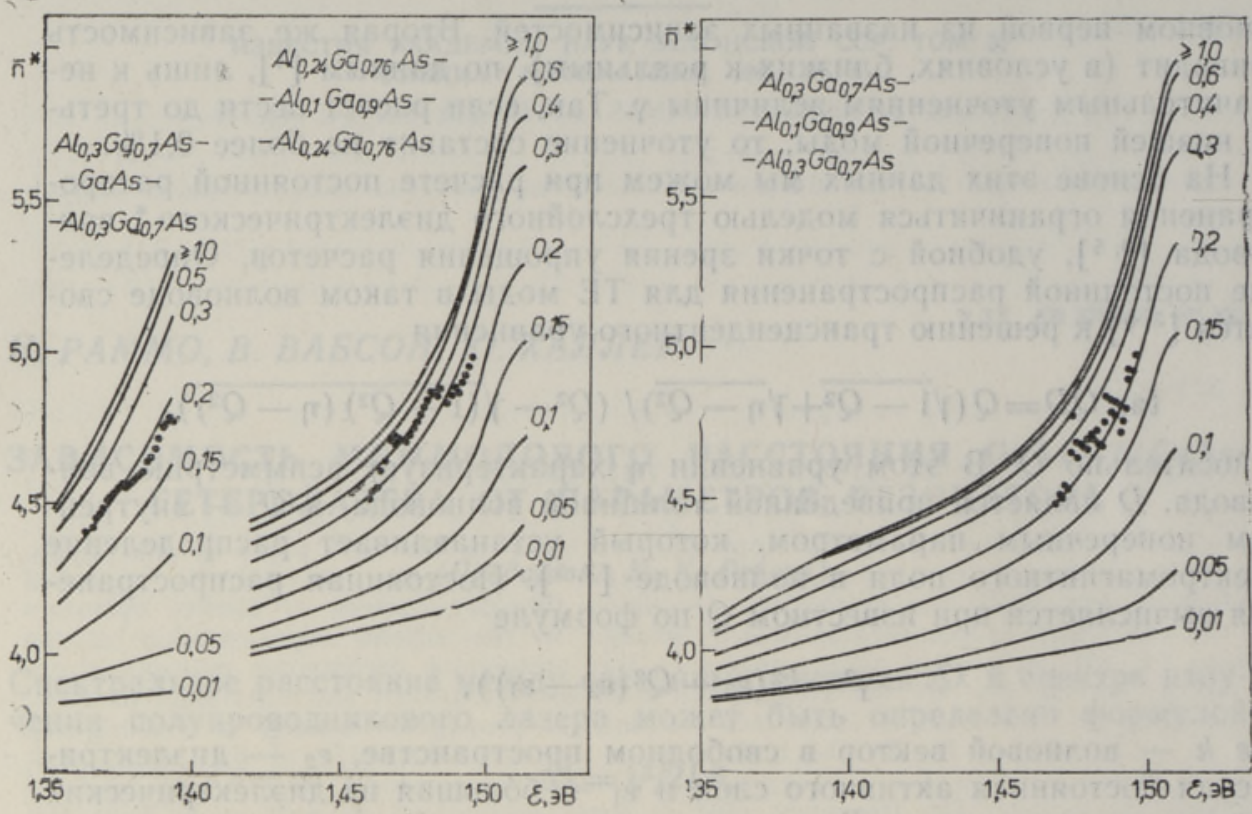

Рис. 1 и 2. Зависимость ЭПП гетеролазеров от энергии кванта излучения при различных толщинах (указанных в мкм) активного слоя. Верхние кривые характеризуют также материал активного слоя, а нижние материал эмиттера. Точками отмечены экспериментально полученные данные.

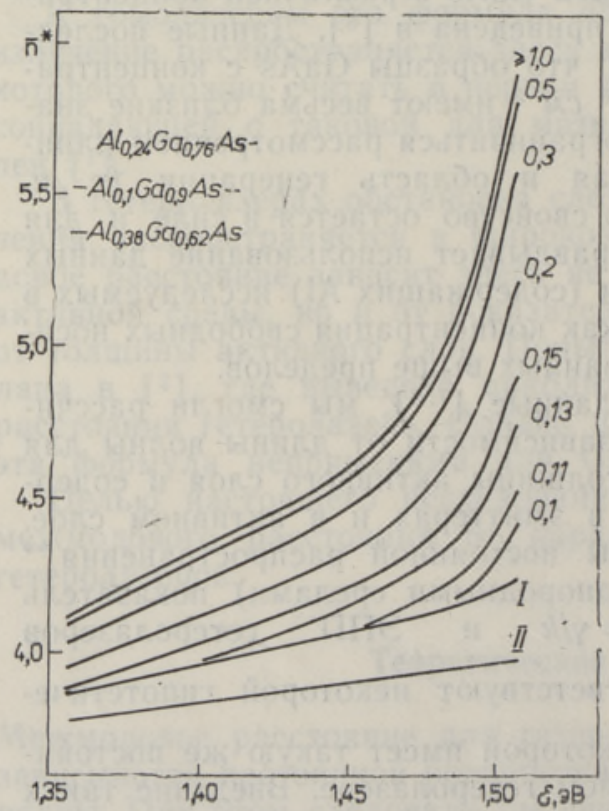

Рис. 3. Зависимость ЭПП гетеролазера от энергии кванта излучения при различных толщинах (указанных в мкм) активного слоя. Верхняя кривая характеризует также материал активного слоя, а кривые I и II - материал эмиттеров $\mathrm{Al}_{0,24} \mathrm{Ga}_{0,76} \mathrm{As}$ и $\mathrm{Al}_{0,36} \mathrm{Ga}_{0,62} \mathrm{As}$ соответственно.

На рис. $1-3$ показаны рассчитанные нами четыре примера зависимости ӘПП гетеролазеров от энергии кванта излучения для нулевой TE моды при различных толщинах активного слоя. Кривые слева на рис. 1 и на рис. 2 иллюстрируют влияние изменения состава активного слоя, а кривые справа на рис. 1 и на рис. 2 влияние изменения состава эмиттеров на ӘПП гетеролазера. На рис. 3 нанесены кривые для несим-

метричного гетеролазера. Из рисунков видно, что при большой толщине $(d \geqslant 1,0$ мкм) активного слоя ЭПП гетеролазеров совпадает с таковым для материала активного слоя, а при уменышении толщины активного слоя приближается к ЭПП материала того эмиттера, для которого эта величина больше, 

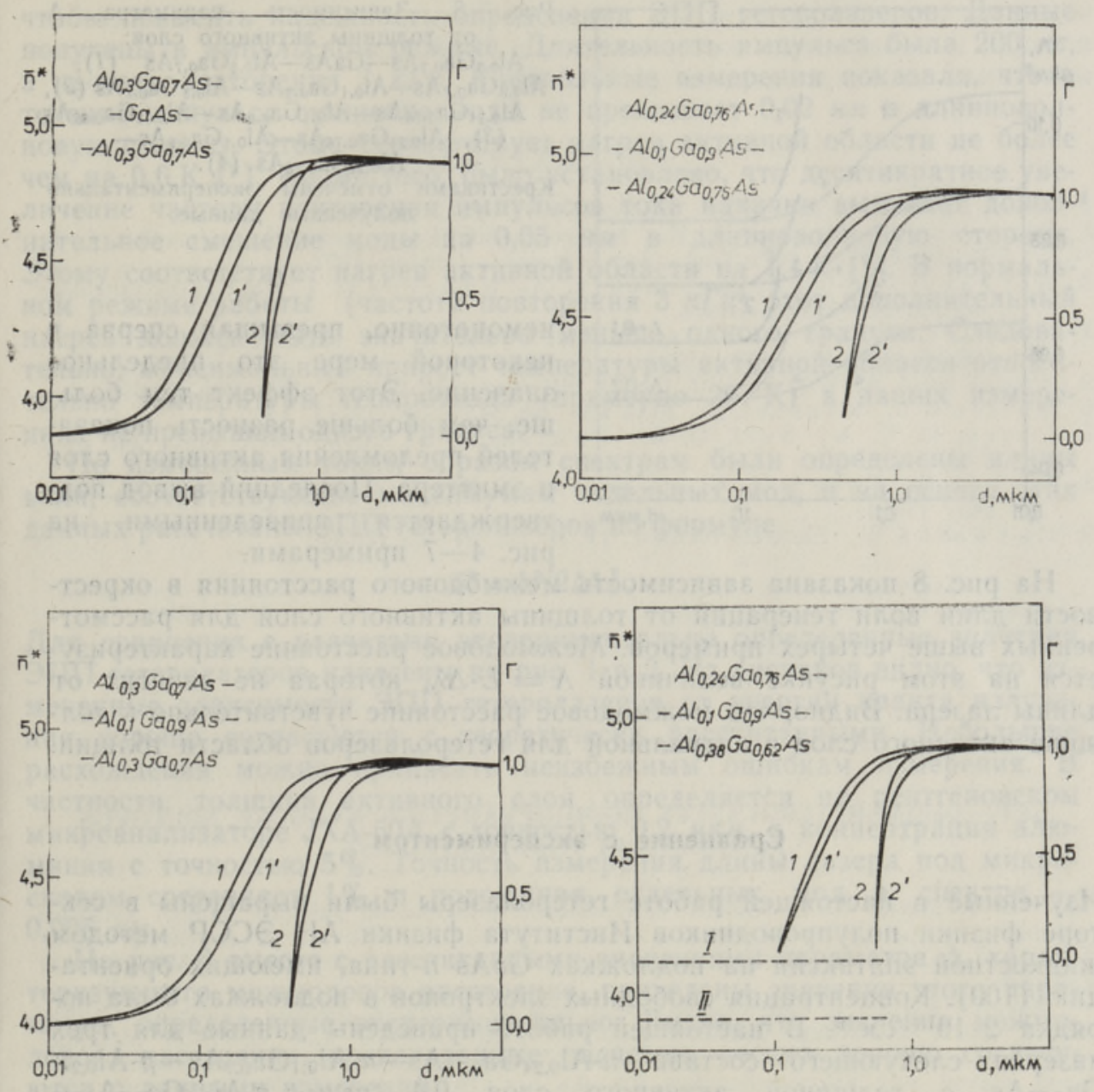

Рис. 4-7. Зависимость ЭПП гетеролазера $(1,2)$ и параметра оптического ограничения $\Gamma\left(I^{\prime}, 2^{\prime}\right)$ от толщины активного слоя для двух первых ТЕ мод на длинах волны генерации. Кривые I и II характеризуют материал эмиттеров $\mathrm{Al}_{0,24} \mathrm{Ga}_{0,76} \mathrm{As}$ и $\mathrm{Al}_{0,38} \mathrm{Ga}_{0,62} \mathrm{As}$ соответственно.

На рис. 4-7 показана зависимость ЭПП гетеролазера от толщины активного слоя (кривые 1 и 2) для двух первых ТЕ мод на длинах волны генерации ***. Эти зависимости показывают, что область быстрого изменения $\bar{n}^{*}$ находится в актуальном для гетеролазеров интервале толщин активного слоя. Для сравнения на рис. 4-7 показаны также зависимости параметра оптического ограничения $\Gamma$ (равного доле оптического потока, заключенной в пределах активного слоя) от толщины активного слоя (кривые $1^{\prime}$ и $2^{\prime}$ ) при тех же длинах волн. Сравнение соответствующих кривых показывает, что изменение ЭПП гетеролазеров определяется в основном изменением параметра оптического ограничения.

Однако нельзя не заметить, что при больших толщинах активного слоя ЭПП гетеролазеров приближается к своему верхнему пределу

*** Для активной области из $\mathrm{GaA}$ s длина волны генерации принята равной 1,375 э $B$, а для активного слоя из $\mathrm{Al}_{0,1} \mathrm{Ga}_{0,9} \mathrm{As}-1,475$ эВ. 


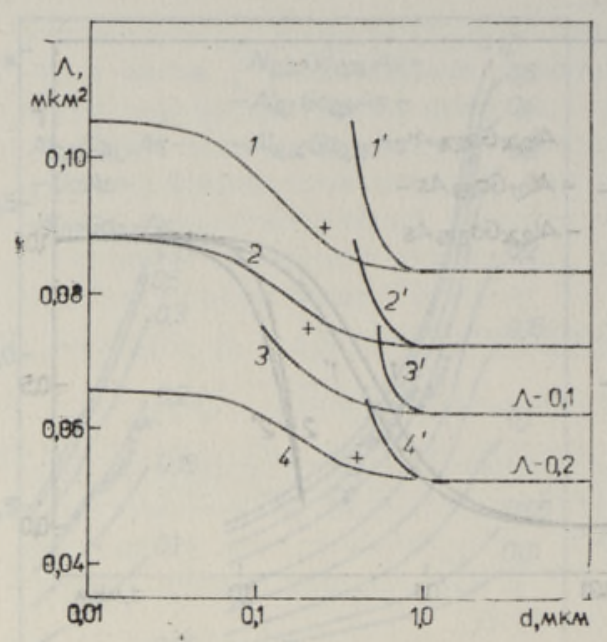

Рис. 8. Зависимость параметра А от толщины активного слоя:

$\mathrm{Al}_{0,3} \mathrm{Ga}_{0,7} \mathrm{As}-\mathrm{GaAs}-\mathrm{Al}_{0,3} \mathrm{Ga}_{0,7} \mathrm{As}$ (1),

$\mathrm{Al}_{0,3} \mathrm{Ga}_{0,7} \mathrm{As}-\mathrm{Al}_{0,1} \mathrm{Ga}_{0,9} \mathrm{As}-\mathrm{Al}_{0,3} \mathrm{Ga}_{0,7} \mathrm{As}$ (2), $\mathrm{Al}_{0,24} \mathrm{Ga}_{0,76} \mathrm{As}-\mathrm{Al}_{0,1} \mathrm{Ga}_{0,9} \mathrm{As}-\mathrm{Al}_{0,38} \mathrm{Ga}_{0,62} \mathrm{As}$ (3), $\mathrm{Al}_{0,24} \mathrm{Ga}_{0,76} \mathrm{As}-\mathrm{Al}_{0,1} \mathrm{Ga}_{0,9} \mathrm{As}$ $\mathrm{Al}_{0,24} \mathrm{Ga}_{0,76} \mathrm{As}$ (4).

Крестиками отмечены экспергмснтально полученные данные.

немонотонно, превышая сперва в некоторой мере это предельное значение. Этот эффект тем больше, чем больше разность показателей преломления активного слоя и эмиттера. Последний вывод подтверждается приведенными на рис. 4-7 примерами.

На рис. 8 показана зависимость межмодового расстояния в окрестности длин волн генерации от толщины активного слоя для рассмотренных выше четырех примеров. Межмодовое расстояние характеризуется на этом рисунке величиной $\Lambda=L \cdot \Delta \lambda$, которая не зависит от длины лазера. Видно, что межмодовое расстояние чувствительно к толщине активного слоя в актуальной для гетеролазеров области толщин.

\section{Сравнение с экспериментом}

Изученные в настоящей работе гетеролазеры были выращены в секторе физики полупроводников Института физики АН ЭССР методом жидкостной эпитаксии на подложках GaAs n-типа, имеющих ориентацию (100). Концентрация свободных электронов в подложках была порядка $2 \cdot 10^{18} \mathrm{~cm}^{-3}$. В настоящей работе приведены данные для трех лазеров следующего состава $n-\mathrm{Al}_{0,24} \mathrm{Ga}_{0,76} \mathrm{As}-n-\mathrm{Al}_{0,1} \mathrm{Ga}_{0,9} \mathrm{As}-p-\mathrm{Al}_{0,24^{-}}$ $\mathrm{Ga}_{0,76} \mathrm{As}$ с толщиной активного слоя $0,4 \quad \mu \kappa м ; n-\mathrm{Al}_{0,3} \mathrm{Ga}_{0,7} \mathrm{As}-$ $n-\mathrm{Al}_{0,1} \mathrm{Ga}_{0,9} \mathrm{As}-p-\mathrm{Al}_{0,3} \mathrm{Ga}_{0,7} \mathrm{As}$ с толшиной активного слоя 0,2 мкм и $n-\mathrm{Al}_{0,3} \mathrm{Ga}_{0,7} \mathrm{As}-p-\mathrm{GaAs}=p-\mathrm{Al}_{0,3} \mathrm{Ga}_{0,7} \mathrm{As}$ с толщиной активного слоя 0,25 мкм ****. В исследуемых лазерах на $p$-эмиттер наращивался контактный слой из GaAs $p$-типа. Ограничение тока в плоскости $p$ - $n$-перехода осуществлялось в первых двух лазерах пленкой из $\mathrm{SiO}_{2}$, а в последнем - обратносмещенным $p$ - $n$-переходом, который создавался между контактным слоем и наращенным на нем слоем $\mathrm{Al}_{\mathbf{a}_{7} \mathrm{Ga}_{0,3} \mathrm{As}}$ $n$-типа. Токопроводящие полоски вытравливались химическим способом через маски, изготовленные методом фотолитографии. Отобранные образцы лазеров первого типа генерировали в непрерывном режиме при комнатной температуре. Однако для целей настоящего исследования непрерывный режим работы лазера оказался неудобным, так как при этом происходит заметный разогрев (порядка $50 \mathrm{~K}$ ) активной области.

Спектры излучения описанных гетеролазеров измерялись с помощью монохроматора THR-1500, имеющего решетку с $1800 u т / \mu м$ и обеспечивающего разрешение лучше, чем 0,005 нм. При этих измерениях мы старались получить как можно более длинный участок спектра,

**** Содержание $\mathrm{Al}$ в эмиттерах и толщииу активного слоя определяли на рентrеновском микроанализаторе JXA-50A. 
чтобы повысить надежность определения ӘПП гетеролазеров. Данные получены в импульсном режиме. Длительность импульса была $200 \mathrm{Hc}$, а частота повторения $3 \kappa \Gamma$. Контрольные измерения показали, что в течение импульса смещение моды не превышает 0,02 нм в длинноволновую сторону. Этому соответствует нагрев активной области не более чем на $0,6 \mathrm{~K}\left[{ }^{9}\right]$. Кроме того, было установлено, что десятикратное увеличение частоты повторения импульсов тока накачки вызывает дополнительное смещение моды на 0,05 нм в длинноволновую сторону. Этому соответствует нагрев активной области на $1,4 \mathrm{~K}\left[{ }^{9}\right]$. В нормальном режиме работы (частота повторения $3 \kappa \Gamma ц$ ) этот дополнительный нагрев должен быть значительно меньше одного градуса. Следовательно, максимальный прирост температуры активной области относительно температуры теплоотвода (примерно $297 \mathrm{~K}$ ) в наших измерениях не превышал одного градуса.

По измеренным таким образом спектрам были определены длины волн, соответствующие максимумам отдельных мод, и на основе этих данных рассчитаны ЭПП гетеролазеров по формуле

$$
\bar{n}^{*}=\lambda^{2} / 2 \Delta \lambda L \text {. }
$$

Для сравнения с расчетами экспериментально определенные значения ЭПП гетеролазеров нанесены на рис. 1 и 2. Из рисунков видно, что измеренные зависимости ЭПП гетеролазеров от энергии кванта излучения хорошо согласуются с теоретически рассчитанными. Небольшие расхождения можно приписать неизбежным ошибкам измерения. В частности, толщина активного слоя определяется на рентгеновском микроанализаторе JXA-50A с точностью 0,2 мкм, а концентрация алюминия с точностью $5 \%$. Точность измерения длины лазера под микроскопом составляет $1 \%$ и положения отдельных мод в спектре $0,005 \mathrm{HM}$.

На рис. 8 вместе с рассчитанными значениями параметра $\Lambda$, характеризующего межмодовое расстояние, приведены значения этого параметра, определенные экспериментально. Видно, что. значения межмодового расстояния, предсказанные расчетом, весьма хорошо согласуются с данными измерений.

\section{Выводы}

1. ЭПП гетеролазеров и межмодовое расстояние зависят не только от состава активного слоя, но и в значительной мере от состава эмиттеров и толщины активного слоя.

2. ЭПП GaAs-AlGaAs-гетеролазеров и межмодовое расстояние меняются от одного предельного значения до другого в актуальном для гетеролазеров интервале толщин активного слоя $(0,1-0,8$ мкм).

3. Зависимость ЭПП гетеролазеров от толщины активного слоя определяется в основном параметром оптического ограничения.

4. Расчетом установлен немонотонный ход ЭПП гетеролазеров при приближении к своему верхнему пределу при увеличении ширины активного слоя.

5. Экспериментально определенные ЭПП гетеролазеров и межмодовое расстояние хорошо согласуются с теоретически рассчитанными.

Авторы выражают благодарность П. Лыуку за полезные заме- 
чания при чтении рукописи, а также Э. Карм, Х. Куус и Р. Каряне за помощь в проведении экспериментов и Е. Асари, Х. Келле за данные измерения на микроанализаторе JXA-50A.

\section{ЛИТЕ РАТ У РА}

1. Marple, T. F., J. Appl. Phys., 35, № 4, 1241-1242 (1964).

2. Kresse1, H., Butler, J., K., Semiconductor Lasers and Heterojunction Leds, Academic Press, New York-San Francisco-London, 1977.

3. Богданкевич О. В., Д а рзнек С. А., Елисеев П. Г., Полупроводниковые лазеры, М., «Наука», 1976.

4. Streifer, W., Don Scif res, R., Burnham, R. D., IEEE J. Quant. Electron., QE-14, № 6, 418-427 (1978).

5. Anderson, W. W., IEEE J. Quant. Electron., QE-1, № 6, 228-236 (1965).

6. Butler, J. K., Kresse1, H., RCA Rev., 38, 542-558 (1977).

7. Casey, H. C. Jr., S el1, D. D., P a nish, M. B., Appl. Phys. Lett., 24, № 2, 63-65 (1974).

8. Sel1, D. D., C a sey, H. C. Jr., Wecht, K. W., J. Appl. Phys., 45, № 6, 26502657 (1974).

9. N a kw a ski, W., Electron Technol., 11, № 1/2, 37-55 (1978).

Ннститут физики

Академии наук Эстонской ССР
Поступила в редакцию 3/VII 1980

\section{RAMMO, V. VABSON, J. HALLER}

\section{GaAs-GaAIAs-HETEROLASERI MOODIDEVAHELISE KAUGUSE SOLTUVUS RESONAATORI PARAMEETRITEST}

Töös on arvutatud GaAs-GaAlAs-heterolaserite pikimoodide vaheline kaugus sōltuvalt lainepikkusest ja aktiivala paksusest erineva koostisega aktiivala ja emitterite korral. Tulemused on esitatud põhiliselt heterolaseri efektiivse murdumisnäitaja (EMN) mõistet kasutades.

Arvutus näitab, et heterolaseri EMN muutub aktiivala paksuse kasvades emitteri aine EMN-ist (suuremat väärtust omavast) kuni aktiivala aine EMN-ini. Moodidevaheline kaugus aga väheneb samal ajal pöördvõrdeliselt EMN-iga. Nimetatud muutused leiavad aset paksusvahemikus $0,1-0,8 \mu \mathrm{m}$. Heterolaseri EMN läheneb oma ülemisele piirväärtusele mittemonotoonselt, läbides väikese maksimumi.

Eksperimendi tulemused on heas kooskōlas arvutustega.

I. RAMMO, V. VABSON, J. HALLER

\section{THE DEPENDENCE OF AXIAL MODE WAVELENGTH SEPARATION ON CAVITY PARAMETERS}

Axial mode wavelength separation of GaAs-GaAlAs-double-heterojunction (DH) lasers versus quantum energy and active layer thickness is numerically calculated for different aluminum-containing active regions and confining layers (refractive index). The results of the calculations are presented using mainly the term of effective refractive index (ERI) of DH lasers.

The calculations indicate that the ERI of DH lasers vary between the ERI of confining layer material (with higher refractive index) and that of the active region material as the active layer thickness increases. The axial mode wavelength separation is inversely proportional to the ERI of DH lasers. These changes of the ERI of DH lasers occur in the active layer thickness interval of 0.1 and $0.8 \mu \mathrm{m}$, actual for DH lasers. The ERI of DH lasers does not approach its limit (at a very thick active region) monotonously but through a slight maximum.

The experimental data are in good agreement with the results of calculations. 